\title{
Correction
}

\section{Correction: Sakrikar et al., Attention Deficit/Hyperactivity Disorder-Derived Coding Variation in the Dopamine Transporter Disrupts Microdomain Targeting and Trafficking Regulation}

In the article "Attention Deficit/Hyperactivity Disorder-Derived Coding Variation in the Dopamine Transporter Disrupts Microdomain Targeting and Trafficking Regulation" by Dhananjay Sakrikar, Michelle S. Mazei-Robison, Marc A. Mergy, Nathan W. Richtand, Qiao Han, Peter J. Hamilton, Erica Bowton, Aurelio Galli, Jeremy Veenstra-VanderWeele, Michael Gill, and Randy D. Blakely, which appeared on pages 5385-5397 of the April 18, 2012 issue, the gene for DAT was incorrectly identified as SLC6A4 on pages 5385 and 5387. The correct identifier is $S L C 6 A 3$. This correction does not affect any conclusion or interpretation. The gene for DAT has also been corrected on the online PDF version.

DOI:10.1523/JNEUROSCI.4296-12.2012 